Paper to be presented at the Proceedings of International Conference on Thermoelectrics, August 25-27, 1997, Dresden, Germany

\section{RARE EARTH THERMOELECTRICS}

G. D. Mahan
RECERPER

OCT 03397

OSTI

Department of Physics, University of Tennessee

Knoxville, TN 37996

and

SOLID STATE DIVISION

OAK RIDGE NATIONAL LABORATORY

Managed by

LOCKHEED MARTIN ENERGY RESEARCH CORP.

Under

Contract No. DE-AC05-96OR22464

With the

U. S. DEPARTMENT OF ENERGY

OAK RIDGE, TENNESSEE

September 1997

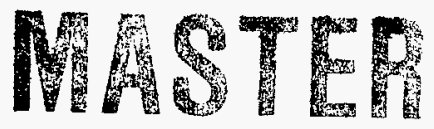




\title{
Rare Earth Thermoelectrics
}

\author{
G.D. Mahan \\ Department of Physics and Astronomy \\ University of Tennessee, Knoxville, TN 37996-1200, and \\ Solid State Division, Oak Ridge National Laboratory \\ Oak Ridge, TN 37831-6030
}

\begin{abstract}
We review the thermoelectric properties of metallic compounds which contain rare-earth atoms. They are the group of metals with the largest value ever reported of the Seebeck coefficient. An increase by $50 \%$ of the Seebeck would make these compounds useful for thermoelectric devices. The largest Seebeck coefficient is found for compounds of cerium (e.g., $\mathrm{CePd}_{3}$ ) and ytterbium (e.g., $\mathrm{YbAl}_{3}$ ). Theoretical predictions are in agreement with the maximum observed Seebeck. We discuss the theoretical model which has been used to calculate the Seebeck coefficient. We are solving this model for other configurations $(4 f)^{n}$ of rare-earth ground states.
\end{abstract}

\section{Introduction}

There is a need to find new materials suitable for thermoelectric applications [ 1-5]. Present materials are good at room temperature and above. New materials at high temperature are always useful for power generation devices. There is also a need for new materials at low temperatures for refrigeration. They would be used in cooling for superconducting magnets and electronics. Generally, a good thermoelectric material must have high values of the electrical conductivity and Seebeck coefficient, while having a small value of the thermal conductivity.

Most metals are unsuitable for thermoelectric applications because they have a small Seebeck coefficient[ 6]. Metallic compounds containing rare-earth compounds are an exception to this rule-of-thumb [7]. The highest values of the Seebeck coefficient are obtained in rareearth compounds of cerium or ytterbium $[5,8]$ such as $\mathrm{YbAl}_{3}$ or $\mathrm{CePd}_{3}$ where $|S| \sim 100 \mu \mathrm{V} / \mathrm{K}$. Some of the materials with high value of the Seebeck and power factor are shown in table 1 . We also include bismuth telluride for comparison.

\begin{tabular}{r|r|rr|rr|c}
\hline \hline Compound & $T(\mathrm{~K})$ & $\mathrm{S}$ & ref. & $\rho$ & ref. & $\mathrm{P}_{f}$ \\
\hline $\mathrm{Bi}_{2} \mathrm{Te}_{3}$ & 300 & 240 & & 1700 & & 35 \\
& & & & & & \\
$\mathrm{CeSn}_{3}$ & 150 & 50 & {$[9-11\}$} & 25 & {$[9,12]$} & 100 \\
$\mathrm{CePd}_{3}$ & 140 & 125 & {$[13,14]$} & 170 & {$[13,14]$} & 92 \\
$\mathrm{YbPd}$ & 250 & -104 & {$[15]$} & 145 & {$[15]$} & 75 \\
$\mathrm{YbAl}_{3}$ & 300 & -90 & {$[16]$} & 45 & {$[16]$} & 180 \\
\hline \hline
\end{tabular}

TABLE I. Seebeck coefficient $S$ in units of $\mu \mathrm{V} / \mathrm{K}$, resistivity $\rho$ in units of $\mu \Omega \mathrm{cm}$, and Power factor $\left(\mathrm{P}_{f}=S^{2} / \rho\right)$ in units of $\mu \mathrm{W} /\left(\mathrm{K}^{2} \mathrm{~cm}\right)$.
These are the highest values of the Seebeck coefficient ever reported for metals. Also note the large power factors compared to bismuth telluride. Unfortunately the large value of the thermal conductivity prevents these materials from having large values of the figure of merit $Z$. $\mathrm{CePd}_{3}$ has a value of $Z T \approx 0.4$. Many other compounds containing $\mathrm{Ce}$ or $\mathrm{Yb}$ have been found to have values of the Seebeck coefficient which are lower $[5,8]$ than those in table 1.

The large value of the thermal conductivity in these materials is due to thermal conduction by electrons. This contribution is given accurately by the Wiedemann-Franz law $[17,18]$ which relates the electronic part of the thermal conductivity $K_{e}$ to the electrical conductivity $\sigma$

$$
K_{e}=\frac{\pi^{2}}{3} \sigma T\left(\frac{k_{B}}{e}\right)^{2}
$$

The W-F law can be used to prove an inportant inequality for the figure of merit. Since everything in the denominator of the formula for $Z T$ is positive

$$
\begin{aligned}
Z T & =\frac{\sigma T S^{2}}{K_{e}+K_{\ell}}<\frac{\sigma T S^{2}}{K_{e}} \\
Z T & <\left(\frac{S}{S_{0}}\right)^{2} \\
S_{0} & =\frac{\pi}{\sqrt{3}}\left(\frac{k_{B}}{e}\right)=156 \mu \mathrm{V} / \mathrm{K}
\end{aligned}
$$

where we used the W-F law in arriving at eqn. (3). Both theory [19] and experiment [20] suggest that the W-F law is valid in rare-earth compounds. However, experimental data [21,22] shows that the phonon part of the thermal conductivity is relatively large at low temperature. Thus (2) is really an inequality, and the Seebeck needs to be larger than $S_{0}$ at low temperatures in order that $Z T \geq 1$.

The large value of the Seebeck coefficient in $\mathrm{Ce}$ and $\mathrm{Yb}$ compounds is caused by the Kondo resonance[23]. This resonance is a sharp feature in the density of states of the conduction electrons at energies very near to the chemical potential. This feature appears to be a delta function when plotted on a large energy scale. However, when plotted on a fine energy scale, one finds that the Kondo resonance has an energy width $\Delta E=k_{B} T_{K}$, where $k_{B}$ is Boltzmanns constant and $T_{K}$ is the Kondo temperature. The actual method of calculating the Kondo resonance, and temperature, are described in the next section. When 


\section{DISCLAIMER}

This report was prepared as an account of work sponsored by an agency of the United States Government. Neither the United States Government nor any agency thereof, nor any of their employees, make any warranty, express or implied, or assumes any legal liability or responsibility for the accuracy, completeness, or usefulness of any information, apparatus, product, or process disclosed, or represents that its use would not infringe privately owned rights. Reference herein to any specific commercial product, process, or service by trade name, trademark, manufacturer, or otherwise does not necessarily constitute or imply its endorsement, recommendation, or favoring by the United States Government or any agency thereof. The views and opinions of authors expressed herein do not necessarily state or reflect those of the United States Government or any agency thereof. 
calculating the Seebeck coefficient, the Kondo resosnance causes a broad peak in the Seebeck coefficient $S(T)$ as a function of temperature $T$. This broad peak has its maximum at, or at least very close to, the Kondo temperature.

The Kondo resonance is due to spin fluctuations. Most rare-earth ions in metals have a magnetic moment due to the partially occupied $f$-shell. A conduction electron, when scattering from the rare-earth ion, can flip its spin while also flipping the moment of the $f$-electrons. This process causes the resistivity $\rho(T)$ to rise at low temperature, which is called the Kondo effect. Later theoretical work showed that this spin-flip scattering caused the energy resonance near to the chemical potential, which we now call the Kondo resonance. The Kondo resonance, and the high value of the Seebeck coefficient, are caused by the conduction electrons interacting with the spin fluctuations. Most theoretical calculations of this phenomena start from a theoretical model proposed by Anderson[ 39].

Numerous theoretical calculations have solved the Anderson model to obtain the Seebeck coefficient for $\mathrm{Ce}$ or $\mathrm{Yb}$. They generally predict the value of $100 \mu \mathrm{V} / \mathrm{K}$, in agreement with the observed maximum[24- 38]. These values are obtained by four different methods: Gunnarsson and Schönhammer $[24,25]$, the non-crossing approximation [26, 28-30] slave-boson techniques[27], and renormalization group techniques[33-36]. The non-crossing approximation is described below.

An interesting question is whether other rare-earth compounds, besides $\mathrm{Ce}$ or $\mathrm{Yb}$, can have a Seebeck coefficient which is higher than $100 \mu \mathrm{V} / \mathrm{K}$. In talking with experimentalists in this field, most admit that they have not tried very hard to find mixed valent materials composed of other rare-earths, once the $\mathrm{Ce}$ and $\mathrm{Yb}$ compounds became fashionable. However, compounds have been made with other rare-earths, and none show a Seebeck as large as $100 \mu \mathrm{V} / \mathrm{K}$. This raises the interesting question of whether any of them are capable of having a larger Seebeck coefficient than this apparent maximum. Theoretical calculations of energy levels indicate that mixed valent materials are possible with other rare earths. So we have started a series of calculations to calculate the Seebeck coefficient for the general configuration $(4 f)^{n}$ where the occupancy integer spans the range $1 \leq n<14$.

We have used the non-crossing approximation to extend these calculations to all values $n$ of the $f$-orbital occupancy. We solve the single site Anderson model[23] for the case of a level with degeneracy $N_{f}$. We find the Kondo resonance for the case that the ground state has $L$ electrons in the $f$-orbital, where $0<L<N_{f}$. The Seebeck coefficient is calculated as a function of temperature for every value of $L$. We find that the Seebeck coefficient is largest for $L=1$ and declines in value as $L$ increases. This suggests that large Seebeck coefficients are not possible in mixed valent systems besides those of cerium or ytterbium. Generally we find that the Kondo resonance becomes smaller when $L$ is increased.
A realistic calculation of the Seebeck for the configuration $(4 f)^{n}$ requires that one include the various splittings of the $f$-levels due to spin-orbit and other interactions. This has only been done for Ce $[25,26,38]$ which has $L=1$. We are in the process of doing this calculation for $L=2$ which corresponds to Pr. It is difficult to guess the results which will be found when we split the levels. The Seebeck coefficient is sensitive to a number of features of the Kondo resonance, such as the width and aymmetry. Any change in parameters changes both these features, which compete against each other for determining the size of the Seebeck coefficient.

\section{Single-Site Anderson Model}

The most popular theoretical model for describing the rare earth compounds was introduced by P.W. Anderson[ $39,23]$

$$
\begin{aligned}
H & =H_{0}+V \\
H_{0} & =\sum_{k \nu} \varepsilon_{k} C_{k \nu}^{\dagger} C_{k \nu}+\varepsilon_{f} \sum_{\mu} n_{\mu}+U \sum_{\mu>\nu} n_{\mu} n_{\nu} \\
n_{\mu} & =f_{\mu}^{\dagger} f_{\mu} \\
V & =\frac{1}{\sqrt{N}} \sum_{k \nu} V_{k \nu}\left[f_{\nu}^{\dagger} C_{k \nu}+C_{k \nu}^{\dagger} f_{\nu}\right]
\end{aligned}
$$

where $V$ is the hybridization interaction between the band electrons $(k)$ and the localized electrons $f_{\mu}$. It describes processes whereby the conduction electrons hop on and off the localized $f$-shell of the rare-earth. The onsite Coulomb repulsion is $U$ and $\varepsilon_{f}$ is the eigenvalue for a single $f$-electron. The eigenvalue and degeneracy factor $Z_{n}$ for the $f$-electrons are, in the absence of hybridization

$$
\begin{aligned}
& E_{n}=n \varepsilon_{f}+\frac{n(n-1)}{2} U \\
& Z_{n}=\left(\begin{array}{c}
N_{f} \\
n
\end{array}\right)
\end{aligned}
$$

\begin{tabular}{c|c|c|c|c|c|c|c}
\hline \hline $3+$ & $\mathrm{nf}$ & $\mathrm{S}$ & $\mathrm{L}$ & $\mathrm{J}$ & $2 \mathrm{~J}+1$ & $\Delta_{-}$ & $\Delta_{+}$ \\
\hline $\mathrm{Ce}$ & 1 & $1 / 2$ & 3 & $5 / 2$ & 6 & 1.8 & 3.2 \\
$\mathrm{Pr}$ & 2 & 1 & 5 & 4 & 9 & 3.8 & 1.9 \\
$\mathrm{Nd}$ & 3 & $3 / 2$ & 6 & $9 / 2$ & 10 & 5.2 & 1.5 \\
$\mathrm{Pm}$ & 4 & 2 & 6 & 4 & 9 & 5.5 & 1.6 \\
$\mathrm{Sm}$ & 5 & $5 / 2$ & 5 & $5 / 2$ & 6 & 5.4 & 0.3 \\
$\mathrm{Eu}$ & 6 & 3 & 3 & 0 & 1 & 7.9 & \\
$\mathrm{Eu}$ & 7 & $7 / 2$ & 0 & $7 / 2$ & 8 & 1.9 & 8.6 \\
$\mathrm{Gd}$ & 7 & $7 / 2$ & 0 & $7 / 2$ & 8 & 8.5 & 3.7 \\
$\mathrm{~Tb}$ & 8 & 3 & 3 & 6 & 13 & 3.2 & 2.2 \\
$\mathrm{Dy}$ & 9 & $5 / 2$ & 5 & $15 / 2$ & 16 & 4.9 & 1.5 \\
$\mathrm{Ho}$ & 10 & 2 & 6 & 8 & 17 & 5.8 & 1.4 \\
$\mathrm{Er}$ & 11 & $3 / 2$ & 6 & $15 / 2$ & 16 & 5.9 & 1.8 \\
$\mathrm{Tm}$ & 12 & 1 & 5 & 6 & 13 & 5.5 & 1.1 \\
$\mathrm{Yb}$ & 13 & $1 / 2$ & 3 & $7 / 2$ & 8 & 7.4 & 0.9 \\
\hline
\end{tabular}

TABLE II. Ground state degeneracy $(2 \mathrm{~J}+1)$ for mostly $3+$ rare earths ions according to Hund's Rules. Values for $\Delta_{+}$and $\Delta$ - from Herbst and Wilkins $[40]$. 
The ground state degeneracy of a rare-earth ion are given according to Hund's rules. These are shown in table 2. The Anderson model ignores the splittings of the $f$ orbital due to spin-orbit and other factors. It gives the wrong degeneracy $Z_{n}$ instead of $(2 J+1)$. In a future paper we will include these realistic splittings of the $f$ levels. The present calculation is intended to be a solution of the Anderson model as given in eqns (5)-(8).

The eigenvalue expression ( 9 ) is parabolic in the level occupancy $n$. Since $\varepsilon_{f}<0$ then the minimum energy is found near to $n \sim 1 / 2-\varepsilon_{f} / U$. Define $L$ as the value of $n$ which has the lowest value of $E_{n}$. We can increase $L$ by decreasing the value of $\varepsilon_{f}$ towards larger negative values, while keeping $U$ fixed.

Mixed valence occurs when the number of $f$-electrons can flucturate between $n$ and $n \pm 1$. This fluctuation is the origin of the spin fluctuations. The hybridization interaction causes mixing whenever $\left|E_{L}-E_{L \pm 1}\right|$ is a small energy. The standard nomenclature[ 40$]$ is to define the two excitation energies out of the ground state as

$$
\begin{aligned}
\Delta_{-} & =E_{L-1}-E_{L}=-\left[\varepsilon_{f}+(L-1) U\right] \\
\Delta_{+} & =E_{L+1}-E_{L}=\varepsilon_{f}+L U \\
U & =\Delta_{+}+\Delta_{-}
\end{aligned}
$$

Values of $\Delta_{ \pm}$have been calculated for the rare-earth metals in ref. [40] and are shown in table 2. Generally they find one value which is small $(\Delta \sim 1.0 \mathrm{eV})$ while the other is larger so that $U \sim 6-8 \mathrm{eV}$. We assume that similar values apply to the rare-earths when they are in metallic compounds.

We are doing a series of numerical calculations where we keep fixed the important values of $\Delta_{ \pm}=E_{L \pm 1}-E_{L}$, while varying $\varepsilon_{f}=-0.5-U(L-1)$ to change $L$. We choose $\Delta_{-}=0.5 \mathrm{eV}$.

First we discuss the properties of the Hamiltonian $H_{0}$ without the hybridization $V$. This interaction is included in the following section. The Green's function for the $f$ states in the absense of the hybridization term is

$$
\mathcal{G}_{f}\left(i \omega_{m}\right)=-\sum_{\mu} \int_{0}^{\beta} d \tau e^{i \omega_{m} \tau}<T_{\tau} f_{\mu}(\tau) f_{\mu}^{\dagger}>
$$

This can be written exactly for the nonintracting states. We employ the notation $N_{f}=14$ to denote the number of states for a single $f$-electron. The degeneracy of the having $n$-electrons in the $f$-state is $Z_{n}$ as defined above.

$$
\begin{aligned}
\mathcal{G}_{f}\left(i \omega_{m}\right) & =\frac{1}{Z} \sum_{n=0}^{N_{f}} Z_{n} e^{-\beta E_{n}}\left\{\frac{N_{f}-n}{i \omega_{m}+E_{n}-E_{n+1}}\right. \\
& \left.+\frac{n}{i \omega_{m}+E_{n-1}-E_{n}}\right\} \\
A_{f}(\omega) & =-2 \Im\left[G_{f}(\omega)\right] \\
A_{f}(\omega) & =\frac{2 \pi}{Z} \sum_{n=0}^{N_{f}} Z_{n} e^{-\beta E_{n}}\left\{\left(N_{f}-n\right) \delta\left(\omega+E_{n}-E_{n+1}\right)\right.
\end{aligned}
$$


ref.[ 26]. First consider the result for the self-energy in the second-order of perturbation theory

$$
\begin{aligned}
\Sigma_{n}(\omega) & =n S_{n-1}^{(0)}(\omega)+\left(N_{f}-n\right) S_{n+1}^{(0)}(\omega) \\
S_{n+1}^{(0)}(\omega) & =\frac{1}{N} \sum_{k} \frac{V_{k}^{2} n_{k}}{\omega-\left(E_{n+1}-\varepsilon_{k}\right)+i \eta} \\
S_{n-1}^{(0)}(\omega) & =\frac{1}{N} \sum_{k} \frac{V_{k}^{2}\left(1-n_{k}\right)}{\omega-\left(E_{n-1}+\varepsilon_{k}\right)+i \eta}
\end{aligned}
$$

There are two terms. The first term $\left(f^{\dagger} C_{k}\right)$ in $V$ adds an electron to the $f$-level, which changes $n$ to $n+1$, while destroying a band electron in the state $k$. This process is proportional to the occupation number $n_{k}$ of the band electrons. The second term $\left(C_{k}^{\dagger} f\right)$ in $V$ takes an electron out of the $f$ level, which changes $n$ to $n-1$, while adding an electron to the band. This process is proportional to the probability $\left(1-n_{k}\right)$ that the band state is unoccupied.

Arguments of $S^{(0)}$ are Green's functions $G_{n}$ without the self-energies. The "Non-Crossing Approximation "(NCA) $[26,38]$ is to evaluate these Green's functions while including these self-energies. Put the self-energies in the denominators

$$
\begin{aligned}
\Sigma_{n}(\omega) & =n S_{n-1}^{\prime}(\omega)+\left(N_{f}-n\right) S_{n+1}(\omega) \\
S_{n+1}(\omega) & =\frac{1}{N} \sum_{k} V_{k}^{2} n_{k} G_{n+1}\left(\omega+\varepsilon_{k}\right) \\
S_{n-1}^{\prime}(\omega) & =\frac{1}{N} \sum_{k} V_{k}^{2}\left(1-n_{k}\right) G_{n-1}\left(\omega-\varepsilon_{k}\right)
\end{aligned}
$$

The above equations are a self-consistent definition for the self-energies. They are solved by iteration on the computer. We find that self-consistency is obtained only after many iterations.

We assume a Lorentzian density of states

$$
g(\varepsilon)=\frac{2 D}{\varepsilon^{2}+D^{2}}
$$

and place the chemical potential at $\varepsilon=0$. The parameter $D$ is an effective band width and the hybridization width is $\Gamma=\pi g(0) V(0)^{2}=2 \pi V(0)^{2} / D$. In $S_{n}^{\prime}(\omega)$ we can change variables $\varepsilon \rightarrow-\varepsilon$ which now makes $S_{n}^{\prime}=S_{n}$. Thus we actually only evaluate the integrals of the form

$$
S_{n}(\omega)=\frac{\Gamma}{\pi} \int \frac{d \varepsilon}{1+(\varepsilon / D)^{2}} n_{F}(\varepsilon) G_{n}(\omega+\varepsilon)
$$

\section{$f$-electron Green's Function}

The final step is to calculate the spectral function of the $f$-electron in the NCA. We must also include in the thermal averages the feature that the interactions give a spread in energy. Our result is

$$
A_{f}(\omega)=\frac{1}{Z} \sum_{n=0}^{N_{f}} Z_{n} \int \frac{d E}{2 \pi} e^{-\beta E} A_{n}(E)\left\{n A_{n-1}(E-\omega)\right.
$$

$$
\begin{gathered}
\left.+\left(N_{f}-n\right) A_{n+1}(E+\omega)\right\} \\
Z=\sum_{n=0}^{N_{f}} Z_{n} \int \frac{d E}{2 \pi} e^{-\beta E} A_{n}(E)
\end{gathered}
$$

A detailed derivation of these equations is given in refs. $[26,41]$. These equations are similar in spirit to those of ref.[ 26]. They took the limit that $U \rightarrow \infty$ which eliminated all states with $n>1$. They only had the two states of $n=0,1$ in their system and $L=1$. Here we include all of the states with different values of $n$. We fix the value $U=6 \mathrm{eV}$. We choose $\varepsilon_{f}=-0.5-U(L-1)$ (in units of electron Volts) to have a variety of values so that we have different occupancies $L$ in the $f$-state, while keeping fixed the values of $\Delta_{-}=-0.5 \mathrm{eV}, \Delta_{+}=5.5 \mathrm{eV}$. We make an approximation in our numerical solution of retaining only the states with $n=L-1, L, L+1$ in our self-consistent solution. We also choose $N_{f}=6$ in order to compare with previous calculations.

\section{Results and Discussion}

Fig. 1 shows a graph of the spectral function $A_{f}(E)$ as a function of $\mathrm{E}$ (in $\mathrm{eV}$ ) for the case that: $L=1, N_{f}=$ $6, \varepsilon=-0.5 \mathrm{eV}, U=6.0 \mathrm{eV}$ at a temperature $T=50 \mathrm{~K}$. The narrow peak near to zero energy is the Kondo resonance. The two broad peaks, one above and one below zero, are at the energies of $\Delta_{ \pm}$. The Kondo resonance is quite visible.

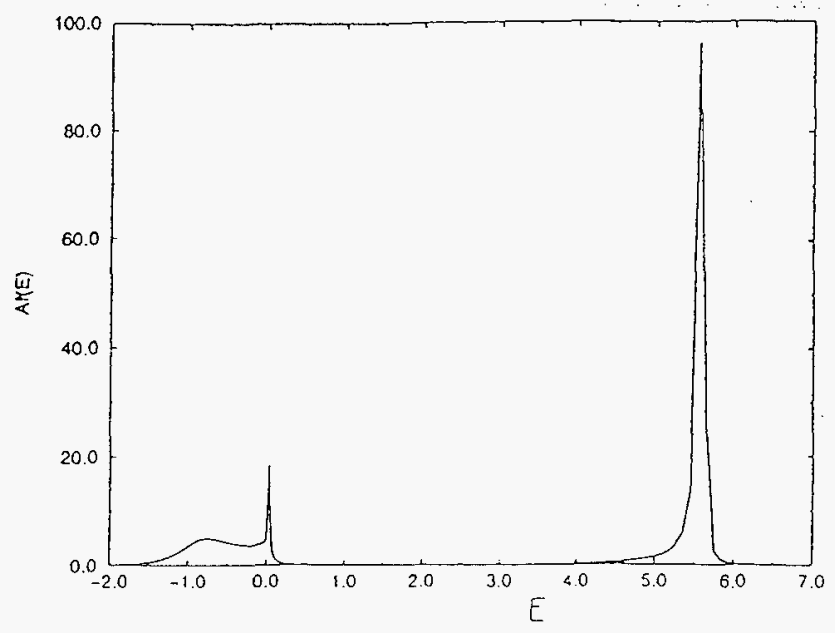

FIG. 1. The spectra function $A_{f}(E)$ of the $f$-electron as a function of energy $E$ in $\mathrm{eV}$. The Kondo resonance appears at zero energy.

The Kondo resonance is a real feature of the density of states of rare-earth compounds. It can be observed directly in photoemission and related spectroscopies, although the results are often controversial[ 42- 47].

We calculated the Kondo resonance in more detail as a function of temperature and $L$. We fixed $N_{f}=6, U, \Delta_{-}$, and $\Delta_{+}$, as described above, and varied the value of $L$ by using $\varepsilon_{f}=-0.5-U(L-1)$. The size of the Kondo resonance decreases in value as $L$ increases. The largest Kondo resonance is the case $L=1$ and the smallest is 
the case with $L=5$. The factors which are changing in these calculations are $\left(n, N_{f}-n\right)$ or $\left(L, N_{f}-L\right)$ in eqns $(27,32)$. These factors relate to the degeneracy of the various levels as given by the factor of $Z_{n}$. If we do a more realistic calculation for $f$-electrons, with the factors which split the levels into sublevels, then we will reduce these factors[ 38$]$.

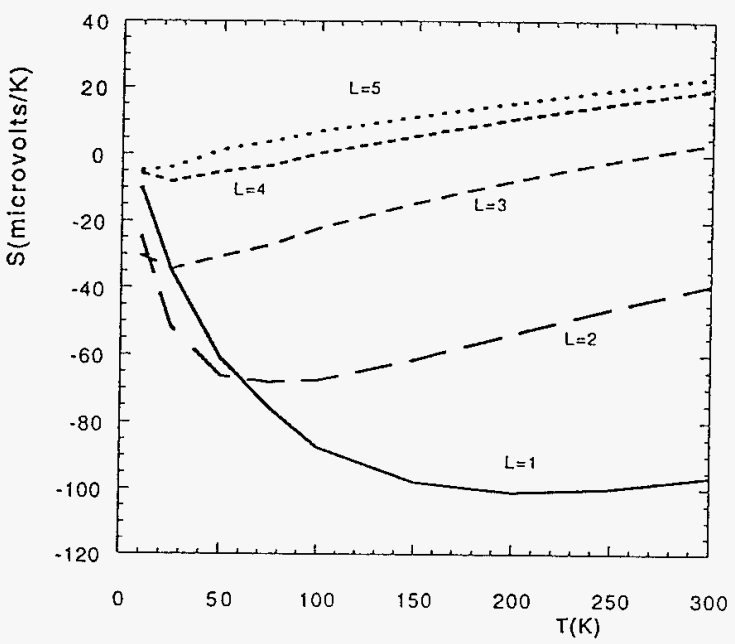

FIG. 2. Seebeck coefficient as a function of temperature between $25 \mathrm{~K}$ and $300 \mathrm{~K}$. The maximum value of the Seebeck coefficient occurs near to the Kondo temperature $T_{K}$. The maximum value of the Seebeck coefficient, and $T_{K}$, both decline with increasing values of $L$. Other parameters the same as in fig. 2 .

Figure 2 shows the Seebeck coefficient for $N_{f}=6$ as a function of temperature for different values of $L$. The Seebeck coefficient is calculated in the usual fashion[ 5 , 26]. Start from the usual formulas

$$
\begin{aligned}
\sigma & =e^{2} \int_{-\infty}^{\infty} d E\left(-\frac{\partial n_{F}(E)}{\partial E}\right) \Sigma(E) \\
T \sigma S & =e \int_{-\infty}^{\infty} d E\left(-\frac{\partial n_{F}(E)}{\partial E}\right) \Sigma(E)(E-\mu) \\
\Sigma(E) & =C \tau(E) \\
\frac{1}{\tau(E)} & =\frac{1}{\tau_{0}}+c A_{f}(E)
\end{aligned}
$$

The factor $\Sigma(E)$ contains the density of states of the band electrons as well as their average velocity and mean free path. We assume that the lifetime $\tau(E)$ has the only significant energy dependence in $\Sigma(E)$, which arises because the band electrons hybridize with the local electrons on the $f$-shells of the rare-earth atoms. The constant lifetime $\tau_{0}$ is from impurity scattering and perhaps phonons. The factors of $c$ and $C$ are constants. Note that it is the inverse of $\Sigma(E)$ which is proportional to $A_{f}(E)$. The Kondo resonance makes a dip in the energy dependence of $\Sigma(E)$.
The above formulas are derived in [5]. The Seebeck coefficient for these cases is shown in fig.(2) as a function of temperature. The largest Seebeck value is for $L=1$, and the values decrease in size when $L$ increases. This agrees with the observation that the Kondo resonance is getting smaller. The Kondo resonance causes the large values of the Seebeck $[5,26]$. The Seebeck coefficient peaks at a temperature near to the Kondo temperature. The peak in our calculated Seebeck coefficients declines with increasing values of $L$. For $L \geq 3$ it is below $50 \mathrm{~K}$ and the peak is off the figure to the left. However, one can see that even at the peak value the Seebeck will be small for these cases. Besides this peak, due to the Kondo resonance, the Seebeck coeficients tends to rise linearly with temperaure, as is typical of metals.

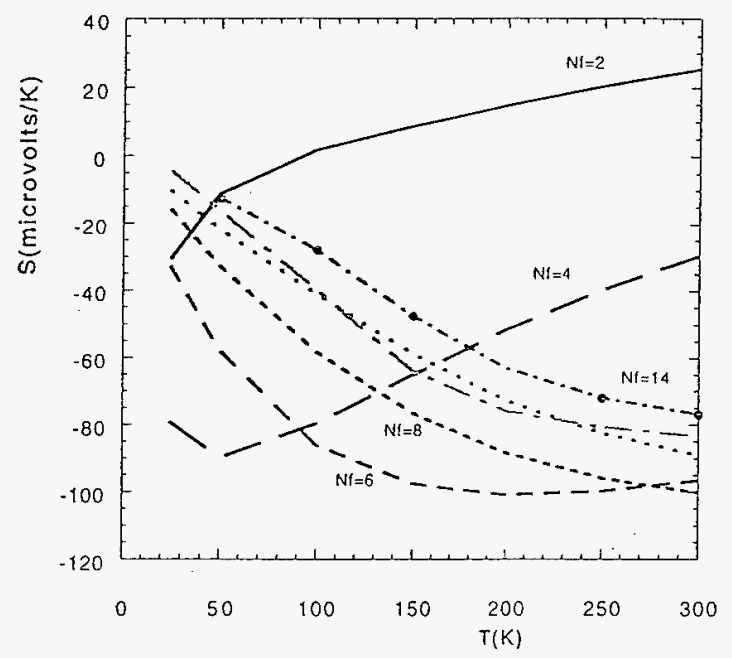

FIG. 3. Calculated Seebeck cofficient as a function of temperature for $L=1, U=6.0 \mathrm{eV}, \Delta_{-}=0.5 \mathrm{eV}, \Delta_{+}=5.5 \mathrm{eV}$ for different values of $N_{f}$.

The Seebeck coefficient has a large value when the Kondo resonance is large and asymmetric. The largest asymmetry is when $L, N_{f}-L$ differ by as much as possible. This happens when $L=1$, or when $L=5$ in the case of $N_{f}=6$. However, because of our choice that $\Delta_{-}$ is smaller than $\Delta_{+}$, the large Kondo resonance happens for $L=1$. If we reverse the magnitudes of $\Delta_{-}$and $\Delta_{+}$ then the largest result is for $L=5$. The mapping of $\Delta_{-} \rightarrow \Delta_{+}, \Delta_{+} \rightarrow \Delta_{-}, L \rightarrow N_{f}-L$ leaves the results unchanged. The case for $\mathrm{Yb}$ corresponds to $N_{f}=8, \Delta_{+}$ small and $\Delta_{-}$large. It will have values similar to those for $N_{f}=6, L=1$ shown in the figures.

Since the largest Seebeck coefficient is for the case of one $f$-electron in the ground state, we were curious how it varied with $N_{f}$. One can choose different values of $N_{f}$, in steps of two, depending upon the crystal field, spin-orbit, or other factors which split the original degeneracy of 14 . These results are shown in figure 3 . Again we used $U=6$ and $\Delta_{-}=0.5$ and $\Delta_{+}=5.5$, all in electron volts. The 
maximum Seebeck is for $N_{f}$ in the range of 6-8, which are the cases of $\mathrm{Ce}$ and $\mathrm{Yb}$, respectively.

So far our calculations show that the Seebeck and Kondo temperature decline in value as one considers values $L$ of $f$-level occupancy larger than one. The same conclusion applies to $f$-holes. Of course, in mixed valence systems, the ground state configuration $(4 f)^{L}$ will change as the occupancy fluctuates. Here we denote $L$ as the principle valence. These results suggest that Ce and $\mathrm{Yb}$ will have the largest values of the Seebeck coefficient, among all of the rare-earth compounds. This theoretical conclusion is in accord with the experimental observations.[ 8].

I wish to acknowledge many helpful conversations with Drs. Brian Sales, Frank DiSalvo, and Mirek Bartkowiak. All have contributed to my education in this field. I also acknowledge research support from a Grant from the Defense Advanced Research Projects Agency, from the University of Tennessee, and from Oak Ridge National Laboratory, managed by Lockheed Martin Energy Research Corp. for the U.S. Department of Energy under contract number DE-AC05-96OR22464.

${ }^{1}$ D.M. Rowe and C.M. Bhandari, Modern Thermoelectrics (Reston, 1983)

${ }^{2}$ H. J. Goldsmid, Electronic Refrigeration (Pion, London, 1986)

${ }^{3}$ C. Wood, "Materials for Thermoelectric Energy Conversion", Rep. Prog. Phys. 51, 459 (1988)

${ }^{4}$ G.A. Slack, in CRC Handbook of Thermoelectrics, ed. D.M. Rowe (CRC Press, 1995) pg 407-440

${ }^{5}$ G.D. Mahan, "Good Thermoelectrics", In Solid State Physics 51, ed. H. Ehrenreich and F. Spaepen (Academic Press, 1998, in press)

${ }^{6}$ F.J. Blatt, P.A. Schroeder, C.L. Foiles, and D. Greig, Thermoelectric Power of Metals (Plenum, 1976)

${ }^{7}$ R.J. Gambino, W.D. Grobman and A.M. Toxen, "Anomalously large thermoelectric cooling figure of merit in the Kondo systems $\mathrm{CePd}_{3}$ and CeIn 3 ", Appl. Phys. Lett. 22, 506 (1973)

${ }^{8}$ D. Jaccard and J. Sierro, "Thermoelectric Power of Some Intermediate Valence Compounds", Valence Instabilities ed. P. Wachter and H. Boppart (North-Holland, 1982) pg 409

9 J. Sakurai, T. Ohyama and Y. Komura, "Thermoelectric Power and Electrical Resistivity of $\mathrm{Ce}\left(\operatorname{In}_{1-x} \mathrm{Sn}_{x}\right)_{3}$, and (Ce $1-x \mathrm{La}_{x} \mathrm{In}_{3}$ ", JMMM 52, 320 (1985)

10 J.R. Cooper, C. Rizzuto, and G. Olcese, "Magnetic Susceptibility, Electronic Specific Heat and Transport Properties of some Intermediate Compounds of Cerium", J. de Phy. C1-32, 1136 (1971)

11 J. Sakurai, H. Kamimura, and Y. Komura, "Thermoelectric Power of $\mathrm{Ce}\left(\mathrm{Pb}_{1-x} \mathrm{Sn}_{x}\right)_{3}$ ",JMMM 76 \& 77, 287 (1988)

${ }^{12}$ B. Stalinski, Z. Kletowski and Z Henkie, "Electrical Resis- tivity of $\mathrm{RESn}_{3}$ Single Crystals ( $\mathrm{RE}=\mathrm{La}, \mathrm{Ce}, \mathrm{Pr}, \mathrm{Nd}$ )", phys. stat. sol.(a) K165 (1973)

13 H. Sthiol, D. Jaccard, and J. Sierro, "Thermoelectric Power of $\mathrm{CePd}_{3+\epsilon} "$, in Valence Instabilities, ed. P. Wachter and H. Boppart (North-Holland, 1982) pg 443

${ }^{14} \mathrm{Y}$. Ijiri and F.J. DiSalvo, "Thermoelectric properties of $\mathrm{R}_{x} \mathrm{Ce}_{1-x} \mathrm{Pd}_{3}\left(\mathrm{R}=\mathrm{Y}, \mathrm{La}_{0.5} \mathrm{Y}_{0.5}, \mathrm{Nd}\right) "$, Phys. Rev. B 55, 1283 (1997)

${ }^{15}$ R. Potts, W. Boksch, G. Leson, B. Politt, H. Schmidt, A. Freimuth, K. Keulerz, J. Langen, G. Neumann, F. Oster, J. Röhler, U. Walter, P. Weidner, and D. Wohlleben, "Magnetic Order and Other Phase Transitions in Mixed-Valent YbPd", Phys. Rev. Lett. 55, 481 (1985)

${ }^{16}$ H.J. van Daal, P.B. van Aken and K.H.J. Buschow, "The Seebeck Coefficient of $\mathrm{YbAl}_{2}$ and $\mathrm{YbAl}_{3}$ ", Phys. Lett. 49A, 246 (1974)

${ }^{17}$ G. Wiedemann and R. Franz, Ann. Phys. 89, 497 (1853)

18 J.M. Ziman, Electrons and Phonons (Clarendon, Oxford, 1960) pg 260

${ }^{19}$ A.K. Bhattacharjee and B. Coqblin, "Thermal Conductivity of cerium compounds", Phys. Rev. B 38, 338 (1988)

${ }^{20}$ E. Bauer, "Thermal Conductivity of $\mathrm{Ce}$ and $\mathrm{Yb}$ Based Kondo Systems", in Transport and Thermal Properties of f-Electron Systems ed. G. Oomi (Plenum, 1993) pg 133

${ }^{21}$ B.C. Sales (private communication)

${ }^{22} \mathrm{C}$. Jones and F. DiSalvo (private communication)

${ }^{23}$ G.D. Mahan, Many-Particle Physics (Plenum, Second Ed., 1990) Chapter 11

${ }^{24} \mathrm{O}$. Gunnarsson and K. Schönhammer, "Double occupancy of the $f$ orbital in the Anderson model for Ce Compounds", Phys. Rev. B 31, 4815 (1995)

${ }^{25} \mathrm{O}$. Gunnarsson and K. Schönhammer, "Many-Body Formulation of Spectra of Mixed Valence Systems", in Handbook on the Physics and Chemistry of Rare Earths, ed K.A. Gschneider, L. Eyring and S. Hüfner (North-Holland, 1987) Vol. 10, pg 103

${ }^{26}$ N.E. Bickers, D.L. Cox and J.W. Wilkins, "Self-Consistent Large-N expansion of Dilute Magnetic Alloys", Phys. Rev. B 36, 2036 (1987)

${ }^{27}$ N.E. Bickers, "Reviews of Techniques in the large-N Expansion for dilute magnetic alloys", Rev. Mod. Phys. 59, 845 (1987)

${ }^{28}$ A. Houghton, N. Read and H. Won, "1/N Expansion for the Transport Coefficients of the Single-Impurity Anderson Model", Phys. Rev. B 35, 5123 (1987)

${ }^{29}$ D.L. Cox and N. Grewe, "Transport Properties of the Anderson Lattice", Z. hys. B 71, 321 (1988)

${ }^{30}$ T.S. Kim and D.L. Cox, "Theory of One-Channel versus Multichannel Kondo Effect for $\mathrm{Ce}^{+3}$ Impurities", Phys. Rev. Lett. 75, 1622 (1995)

${ }^{31}$ H. Schweitzer and G. Czycholl, "Resistivity and Thermopower of Heavy Fermion Systems", Phys. Rev. Lett. 67, 3724 (1991)

${ }^{32}$ H.L. Neal and D.J. Collins, "Transport Coefficients for the Anderson Model", Phys. Rev. B 48, 4299 (1993)

${ }^{33}$ H.O. Frota and L.N. Oliveira, "Photoemission spectroscopy for the spin-degenerate Anderson Model", Phys. Rev. B 33, 7871 (1986)

${ }^{34}$ A. Schiller and V. Zevin, "Degenerate Anderson-impurity Model with Finite On-site Coulomb Interaction", Phys. 
Rev. B 47, 9297 (1993)

${ }^{35}$ T.A. Costi and A.C. Hewson, "Transport Coefficients of the Anderson Model", J. Phys. CM5, L361 (1993)

${ }^{36}$ A.C. Hewson, The Kondo Problem to Heavy Fermions (Cambridge, 1993)

${ }^{37}$ S. Suzuki, O. Sakai and Y. Shimizu, "Magnetic Excitations and Transport Coefficients of the Impurity Anderson Model", J. Phys. Soc. Jpn 65, 4034 (1996)

${ }^{38}$ T.S. Kim and D.L. Cox, "Scaling analysis of a model Hamiltonian for $\mathrm{Ce}^{+3}$ impurities in a cubic metal", Phys. Rev. B 54, 6494 (1996)

${ }^{39}$ P.W. Anderson, "Localized Magnetic States in Metals", Phys. Rev. 124, 41 (1961)

${ }^{40}$ J.F. Herbst and J.W. Wilkins, "Calculation of $4 \mathrm{f}$ Excitation Energies in Metal", in Handbook on the Physics and Chemistry of Rare Earths ed. K.A. Gschneider, L. Eyring and S. Hüfer (North-Holland, 1987) Vol. 10, pg. 321

${ }^{41}$ G.D. Mahan, "Seebeck Coefficient for the Anderson Model", Phys. Rev. B (in press)

${ }^{42} \mathrm{~F}$. Patthey et al, "Characterization of the hybridized $4 f$ states in $\mathrm{YbAl}_{3}$ by high energy spectroscopies", Phys. Rev. B 36, 7697 (1987)

43 S.-J. Oh et al, "Observation of Kondo resonance in $\mathrm{YbAl}_{3}$ ", Phys. Rev. B 37, 2861 (1988)

44 J.-M. Imer et al, "Comment on 'Observation of Kondo resdonance in $\mathrm{YbAl}_{3}$ "', Phys. Rev. B 46, 1864 (1992)

${ }^{45} \mathrm{~S} . J$. Oh et al, "Reply to 'Comment on Observation of Kondo Resonance in $\mathrm{YbAl}_{3}$ "', Phys. Rev. B 46, 1866 (1992)

${ }^{46}$ L.H. Tjeng et al, "Temperature Dependence of the Kondo Resonance in $\mathrm{YbAl}_{3}$ ", Phys. Rev. Lett. 71, 1419 (1993)

${ }^{47}$ R.I.R. Blyth et al, "Temperature-invariant valence band $4 f$ photoemission features in the heavy-fermion compound $\mathrm{YbAl}_{3} "$, Phys. Rev. B 489497 (1993) 
M98000337

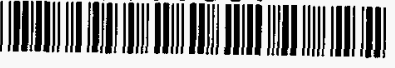

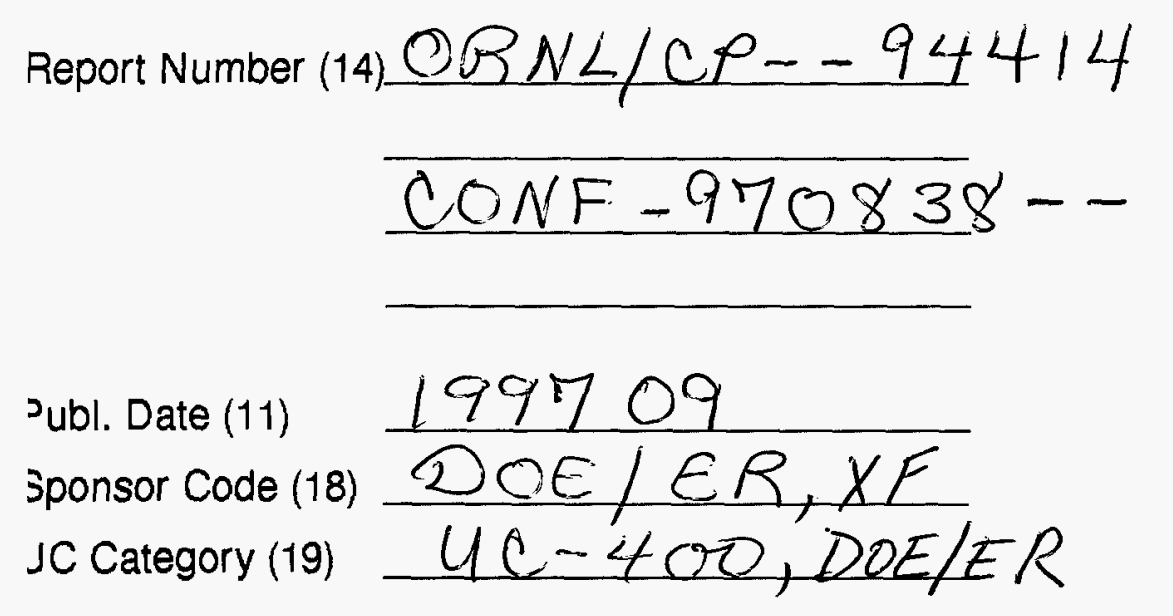

